\title{
Publicité et valeurs socioculturelles implicites : la langue française prostrée à l'image de la saillance*
}

\author{
Aránzazu GIL CASADOMET \\ Universidad Autónoma de Madrid \\ aranzazu.gil@uam.es \\ http://orcid.org/0000-0003-2339-7429
}

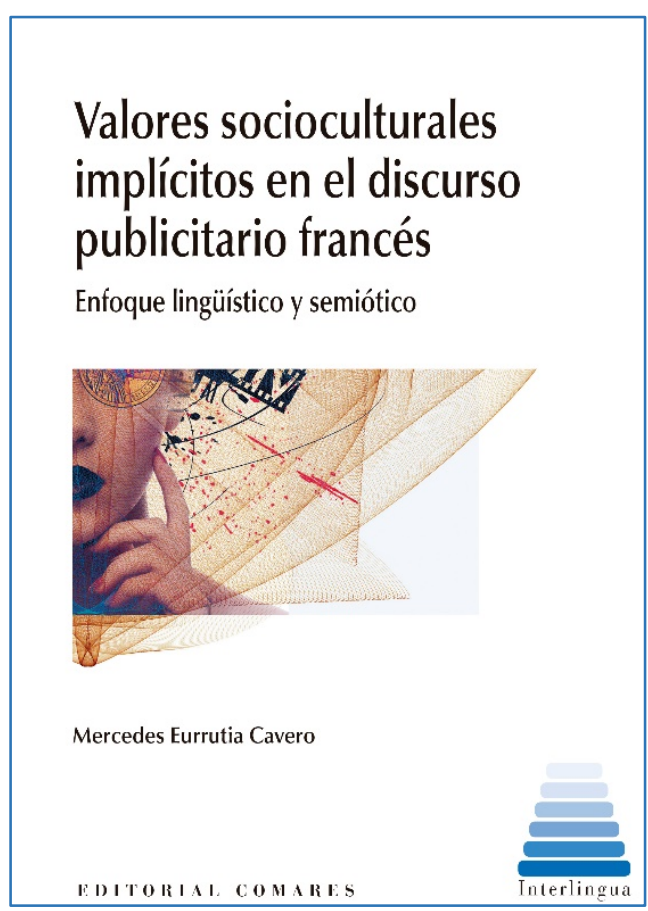

La publicité exerce un rôle psychologique sur un public cible à fins commerciales, ce qui lui rend émettrice de valeurs socioculturelles implicites et garante de la construction identitaire d'une société. Autour de ce sujet, M. ${ }^{a}$ Mercedes Eurrutia Cavero suscite une profonde réflexion dans son ouvrage Valores socioculturales implicitos en el discurso publicitario francés. Enfoque lingüistico y semiótico. Sous la prémisse " telle société, telle publicité » et vice versa (Dayan, 2003 : 74), l'auteure s'interroge ainsi sur les usages linguistiques et le "pouvoir" de l'image comme reflets argumentatifs de la séduction publicitaire. Ce recueil de sept chapitres (quatre rédigés en espagnol et trois en français) a été consacré sous différentes perspectives regroupant la publicité et l'interculturalité, telles que des aspects didactiques qui caractérisent le discours publicitaire, des ressources sémiotiques du discours publicitaire dirigé aux enfants, des valeurs culturelles implicites identitaires d'une nation dans un monde globalisé et leur manifestation linguistique, l'évolution socioculturelle de la femme représentée dans des affiches

\footnotetext{
* Au sujet de l'ouvrage de Mercedes Eurrutia Cavero, Valores socioculturales implícitos en el discurso publicitario francés. Enfoque lingüistico y semiótico (Granada, Editorial Comares, col. «Interlingua», 2021, 196 pages. ISBN : 978-84-1369-115-2).
} 
publicitaires, ainsi que d'autres moyennant le discours touristique promotionnel et la publicité sociale.

Dans le premier chapitre "Publicidad e Interculturalidad ", Eurrutia Cavero montre comment la publicité et la culture révèlent les idéologies implicites existant dans les manifestations sociales, individuelles et interculturelles. Les notions de " culture cultivée » et "culture partagée " y sont incluses (Galisson, 1999), elles correspondent au passage de l'instruction et la connaissance encyclopédique aux traditions, mœurs, valeurs, croyances... des individus et des sociétés. C'est ainsi que la publicité devient un coffre d'outils pour la compréhension et l'analyse de différences interculturelles. En définitive, de l'avis de l'auteure, il s'agit d'un discours onirique qui reflète les modes de vie, les mentalités et les comportements sociaux.

Le deuxième chapitre porte pour titre "Didáctica del discurso publicitario francés en un mundo globalizado: Valores socioculturales implícitos desde un enfique lingüístico comunicativo " et présente le discours publicitaire sous une perspective communicative et sa dimension illocutoire (Austin, 1970 : 137) indirecte et implicite. La saillance persuasive affecte autant aux mots qu'aux entités, codes chromatiques, sons, symboles, etc. Tel qu'Eurrutia Cavero l'explique, tous les individus sont attirés par un élément du message communicatif, en fonction de leurs états affectifs et émotionnels. De surcroît, l'auteure insiste sur les stratégies adoptées concernant les marques des entreprises, comme émettrices des valeurs implicites véhiculant le domaine entrepreneurial et la proximité aux clients; les messages transmis par les produits, comme référents de valeurs idéologiques; et le public cible en tant que récepteurs d'une communication publicitaire qui dans l'actualité est caractérisé par les sociostyles (proposés par le Centre de communication avancé d'Eurocom en 1972). Somme toute, des éléments de base pour l'exploitation didactique des diverses typologies discursives, composantes du discours publicitaire.

Le troisième chapitre "Valores socioculturales implícitos del discurso publicitario dirigido a los niños : Recursos semióticos " permet de prendre conscience de l'emploi des symboles, caractères, logos, langages, etc. dans le but de promouvoir les produits d'achat et comprendre la réaction des enfants face aux marques commerciales dans un processus de communication. D'après l'auteure, il est pertinent de créer de la publicité engagée avec les mineurs pour qu'elle soit un instrument ludique et éducatif de transmission de valeurs socioculturelles. Certaines marques comme Chicco, Petit Suisse, Bimbo, Danonino de Danone, etc. exploitent des personnages adaptés à l'attention des enfants. C'est ainsi qu'une bonne marque doit tenir à stimuler la curiosité et imagination de l'enfant, et pour ce faire, l'entreprise doit baser sa relation avec le public cible en la personnalisation, la pertinence et la permanence du message émis.

Dans le quatrième chapitre, "Manifestación publicitaria de valores culturales implícitos identitarios de una nación en un mundo global ", la diversité culturelle des noms de marque est mise en relief, selon l'auteure, elle "constitue une source de 
richesse et sera l'argument le plus fort contre l'anonymat de la mondialisation et par conséquent, de la terminologie servant à l'exprimer » (p. 48). À l'égard de Charaudeau (1995), il est nommé ici le dit " contrat de communication " en tenant compte que la marque en fait partie et elle est dotée d'une véritable identité établissant une relation avec le consommateur. Dans un sens subjectif, l'image de marque est traitée dans ces pages à l'instar d'une représentation mentale qui dépend du public cible et des effets de mode. Ainsi, des marques telles que Renault, Peugeot, Michelin, etc. projettent d'emblée une image de la France comme nation industrielle, ou une nation conquérante et à la mode d'après les marques Chanel ou L'Oréal. Eurrutia Cavero insiste sur le fait qu'une marque existe grâce à une identité nationale ; afin de le montrer, elle analyse dans ce chapitre plusieurs références culturelles identitaires de la France, sachant que c'est la véritable clef du succès de certaines marques et du rapprochement des sociétés.

Le cinquième chapitre est consacré à l'évolution socio-culturelle de l'image de la femme dans la publicité. L'auteure nous fournit une réflexion de l'évolution historique de cette image dans la société française et une analyse des besoins dans le discours publicitaire sur la femme. Au long des plusieurs décennies, selon ce parcours historique, sont dégagés les différents codes qui créent une sorte de conscience collective sur la femme toujours dans un contexte dynamique de persuasion publicitaire. En ce sens, des ressources linguistiques - figures de style: métaphore, métonymie, synecdoque, hyperbole, antithèse, antiphrase, allitération, détournement - ou sémiotiques - images, symboles, icones, le cadrage, la typographie, les couleurs, les formes - y sont récurrentes. Il va de soi que ces stéréotypes sociaux sont présents dans les stratégies publicitaires axées sur les femmes. La publicité a fait montrer dans ces décennies comment l'image d'une femme ménagère et éducatrice a atteint un référent de femme polyvalente à la recherche de son indépendance personnelle et professionnelle. Eurrutia Cavero se sert de la définition de Gustave Fischer, en guise de conclusion de cette évolution stéréotypée du rôle de la femme dans la société de consommation : "Manières de penser par clichés, qui désignent les catégories descriptives simplifiées basées sur des croyances et des images réductrices par lesquelles nous qualifions d'autres personnes ou d'autres groupes sociaux, objets de préjugés " (Fischer, $1996: 133$ ).

Le titre du sixième chapitre illustre bien le contenu : " Ressources linguistiques et valeurs socioculturelles implicites dans le discours publicitaire français de promotion touristique sur le Web : Substantifs affectifs et évaluatifs». Suivant les théories de Kerbrat-Orecchioni (1998), l'auteure montre une forme de subjectivité évaluative et affective selon la réaction émotionnelle du sujet dans le discours promotionnel touristique. À l'aide des brochures touristiques promotionnelles - catalogues, prospectus, dépliants, feuillet ou encart publicitaires, petit annonce, publipostage, courriel -, Eurrutia Cavero fait l'étude des substantifs en langue française où elle détermine que ceux-ci véhiculent un sens affectif et évaluatif axiologique et non-axiologique, afin de persuader les touristes potentiels et promouvoir une destination touristique. L'analyse du corpus de 
référence mène à l'auteure à la conclusion qu'il est nécessaire de créer une méthode linguistique de calcul de taux de subjectivité des énoncés, permettant le repérage automatique des valeurs évaluatifs, compte tenu de l'existence des valeurs socioculturelles implicites transmises à travers le discours publicitaire.

Dans le septième et dernier chapitre intitulé « Approche linguistique et sémiotique de la publicité sociale française : De la transgression à la pédagogie ", on analyse différentes stratégies communicatives des campagnes de publicité sociale. D'après l'auteure, "À une époque où les valeurs sont en crise, la publicité sociale se substitue à la morale, aux lois socioculturelles. Malgré son but positif, cette publicité de prévention se caractérise par un contenu essentiellement menaçant qui contextualise des images violentes, des réalités sociales déplaisantes volontairement refoulées » (p. 164). Ces stratégies se caractérisent par les facteurs de saillance envers le public cible et liés à la forme et au sens de l'énoncé, de même qu'à l'orientation des images, symboles et signes iconiques que le concepteur de la création publicitaire veut nous transmettre. Ainsi, Eurrutia Cavero montre-t-elle comment la publicité sociale, même étant originaire de la publicité commerciale, vise à influer le comportement du public cible par des moyens de communication persuasive à des fin sociales.

Cet ouvrage constitue, à notre avis, une lecture incontournable abordant des sujets autour des domaines de la publicité, la culture et la linguistique. Reliés d'une manière cohérente, le fil conducteur des sept chapitres donne une vision générale et complète de l'influence de la publicité sur les valeurs socioculturelles et la disposition du message employé par le concepteur des créations publicitaires. En effet, ce recueil retrace un parcours non seulement théorique mais aussi d'analyse de la mise en pratique de deux approches, linguistique et sémiotique. À ce propos, l'auteure invite les récepteurs/trices, lecteurs/trices, curieux/euses de cette publication à faire le point sur une réflexion de la présence de la saillance persuasive dans la publicité commerciale et sociale. Le prétexte sous-entendu dévoile la publicité transmettrice des valeurs socioculturelles implicites, opaques la plupart de fois pour le public cible, mais bien éclaircies lors d'une étude profonde de la problématique.

\section{RÉFÉRENCES BIBLIOGRAPHIQUES}

AUSTIN, John L. (1962, 1970) : Quand dire c'est faire. Paris, Éditions du Seuil.

Charaudeau, Patrick (1995) : «Ce que communiquer veut dire . Sciences Humaines, 51, 45-67.

DAYAN, Armand (2003) : La publicité. Paris, Presses universitaires de France (coll. «Que saisje?»).

FICHER, Gustave. N. (1996) : Les concepts fondamentaux de la psychologie sociale. Paris, Dunod. 
GALISSON, Robert (1999) : "La pragmatique lexiculturelle pour accéder autrement, à une autre culture, par un autre lexique ». ELA, 16, 20-41.

KERBRAT-ORECCHIONI, Catherine (1998) : "La argumentación en la publicidad ». Escritos, Revista del Centro de Ciencias del Lenguaje, 17-18, 291-326. 